\title{
Perturbative vs. Non-Perturbative Scaling Violation in Quark Fragmentation ${ }^{\star}$
}

\author{
J. Engels \\ University of Bielefeld, D-4800 Bielefeld 1, Federal Republic of Germany \\ J. Dabkowski and K. Schilling \\ University of Wuppertal, D-5600 Wuppertal 1, Federal Republic of Germany \\ Received July 20, 1979
}

\begin{abstract}
We investigate the approach to scaling for non-perturbative quark fragmentation in the framework of the uncorrelated jet model. It is found that subasymptotic kinematic scale breaking is comparable in size to scaling violations from hard gluon emission a la QCD. Experimental data available at present do allow for such non-perturbative scale breaking effects.
\end{abstract}

\section{Introduction}

Considerable effort has been spent recently to test the experimental validity of Quantum Chromodynamics (QCD) [1]. In the phenomenological applications of QCD, one discriminates between soft and hard processes. So far, only hard processes can be predicted by the theory, using perturbation methods. The evaluation of soft processes on the other hand is plagued by infrared divergences and a large coupling constant, prohibiting a perturbative treatment [2]. In practice therefore, non-perturbative effects, wherever they matter, are treated in an ad hoc manner. In the case of quark fragmentation into hadrons, e.g., one generally thinks in terms of a quark cascade picture to describe the low $p_{T}$ non-perturbative part of hadron distributions [3]. In general one follows Feynman and Field [3], who proposed a model of this type with built-in scaling. In this framework scaling violations are then considered to be due to hard gluon emission as obtained from AltarelliParisi like equations $[4,5]$.

It is obvious that the relevance of any such test of QCD rests heavily on the validity of one's prejudices about soft physics. If we accept the idea of asymptotic scaling in the non-perturbative quark fragmentation

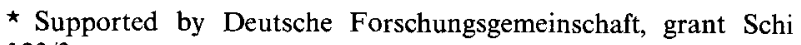
$123 / 3$ into (low $p_{T}$ ) hadrons, is it really reasonable to assume early scaling from the very beginning? In the case of current fragmentation studies in semiinclusive neutrino scattering at SPS energies [6], for example, where the available $Q^{2}$ range is $1 \mathrm{GeV} \lesssim Q^{2} \lesssim$ $100 \mathrm{GeV}^{2}$, this turns out to be a crucial question for any QCD oriented analysis. Naively, one would expect that non-perturbative scaling is only reached, when longitudinal and transverse (with respect to original quark momentum) directions in the final state hadron configuration are clearly distinguished in the sense that $\left\langle p_{\|}\right\rangle \gg\left\langle p_{T}\right\rangle$. This condition is not even fulfilled for the kinematically favoured case of $e^{+} e^{-}$annihilation into hadrons, where $Q^{2}$ values as high as $900 \mathrm{GeV}^{2}$ are nowadays accessible: first data taken at $Q^{2}=289 \mathrm{GeV}^{2}$ by the TASSO detector at PETRA yield an average longitudinal (with respect to the jet axis) momentum of secondaries, which is still of the same order of magnitude as the average transverse momentum, $\left\langle p_{\|}\right\rangle \approx 2.7\left\langle p_{T}\right\rangle=920 \mathrm{MeV}$ [7]. Indeed, subasymptotic scale breaking for the nonperturbative quark fragmentation in $e^{+} e^{-}$annihilation was predicted long ago to occur up to $Q^{2}$ values of around $400 \mathrm{GeV}^{2}$ from an uncorrelated jet model study [8], that was based on input parameters from $p p$ collisions available at that time.

In view of the general interest to extract information about leading order [6,9-12] (and even next to leading order [13]!) QCD effects out of fragmentation studies we think it is necessary to study in more detail than before the problem of the size of subasymptotic scaling violations to be expected from the non-perturbative contribution. To be precise, we want to consider $e^{+} e^{-}$annihilation into pions, using a model with full kinematics but little dynamics. The uncorrelated jet model (UJM) [14], which is nothing else but a transversely limited phase space model, seems to be most appropriate for this purpose, since one would expect any realistic scaling model 


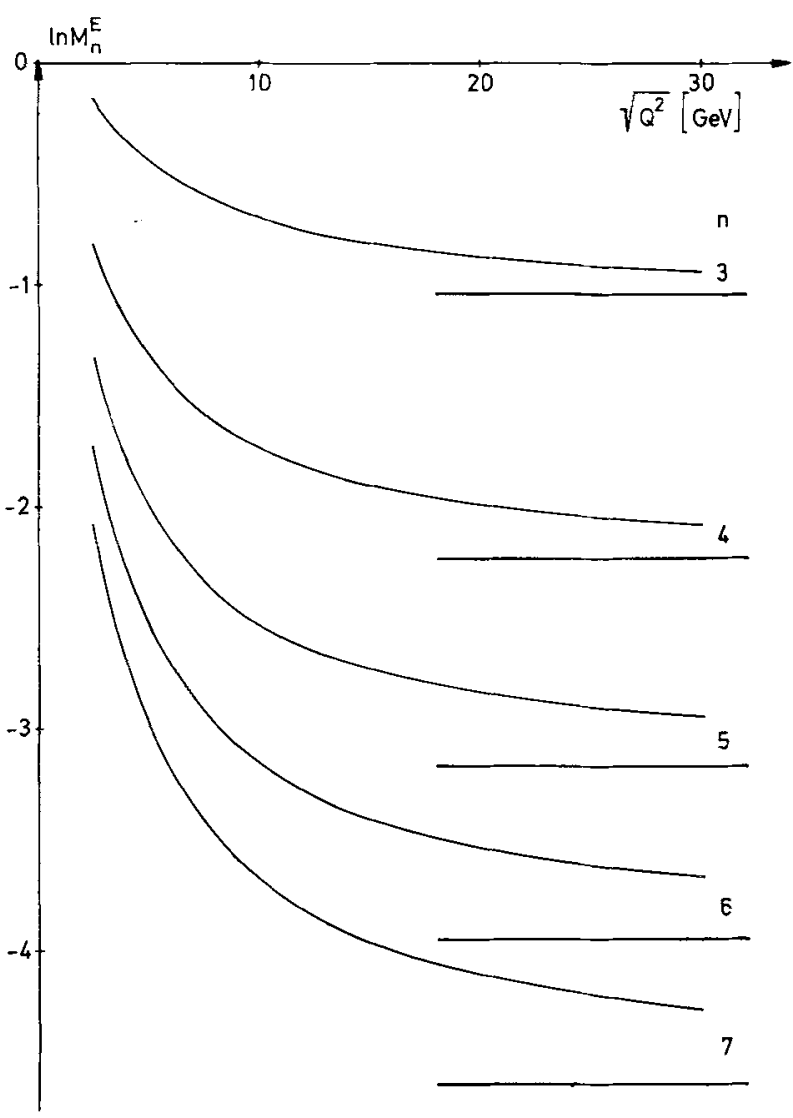

Fig. 1. $\sqrt{Q^{2}}$-dependence of the natural logarithm of moments from non-perturbative quark fragmentation with respect to the scaling variable $x=2 p_{0} / \sqrt{Q^{2}}$, see Eqs. $(9,10)$. The various $n$-values are listed. The horizontal lines indicate the asymptotic scaling limits of these moments

to show such subasymptotic kinematical scale breaking effects.

In Sect. II we shortly remind the reader about the essentials of the uncorrelated jet model. Section III describes and discusses the results. The conclusions are given in Sect. IV.

\section{The Uncorrelated Jet Model}

We imagine the quark pair produced in $e^{+} e^{-}$annihilation to radiate pions ${ }^{1}$ with limited transverse momentum according to the uncorrelated jet model [8]. The fully exclusive distribution for the production of $N$ particles of four-momenta $p_{i}$ in that model is given by

$\Gamma_{N} \sim \frac{y^{N}}{N !} \delta^{(4)}\left(\sum_{j=1}^{N} p_{j}-q\right) \prod_{i=1}^{N} \frac{d^{3} p_{i}}{2 p_{i 0}} f\left(p_{i T}\right)$,

where $q$ is the total four-momentum of the $e^{+} e^{-}$. system and $p_{T}$ is the momentum transverse to the jet

1 It is clear that inclusion of heavier particles [15] would increase the nonasymptotic effects

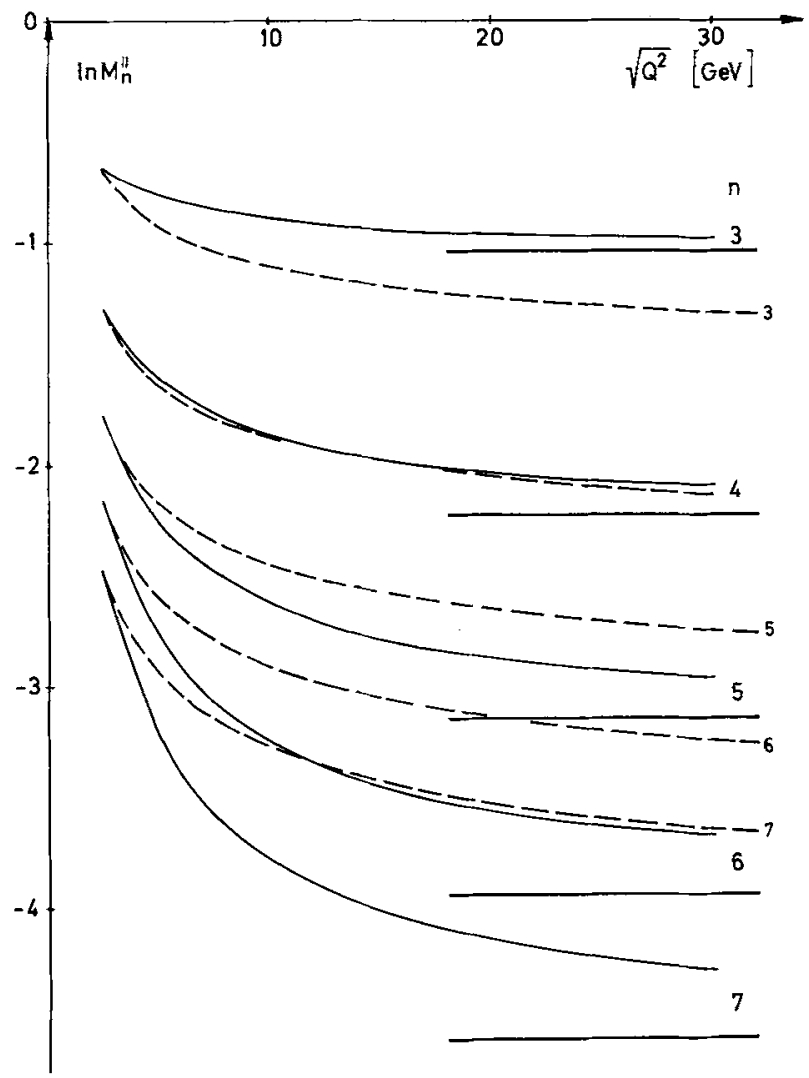

Fig. 2 Same as Fig. 1, but with respect to the variable $x_{\|}=2 p_{\|} / \sqrt{Q^{2}}$. The dashed curves represent the QCD predictions for nonsinglet moments, calculated with $A^{2}=.5 \mathrm{GeV}^{2}$ [5]. Their $Q^{2}$-dependence is characteristic for the amount of scale breaking in QCD

axis. For simplicity we assumed the pions to be chargeless. The function $f\left(p_{T}\right)$ describes the transverse momentum cutoff and is normalized such that

$\pi \int_{0}^{\infty} f\left(p_{T}\right) p_{T} d p_{T}=1$

The total phase-space volume is then given by

$$
\begin{aligned}
& \Omega(q)=\sum_{N=2}^{\infty} \frac{v^{N}}{N !} \int \prod_{i=1}^{N} \frac{d^{3} p_{i}}{2 p_{i 0}} f\left(p_{i T}\right) \delta^{(4)}\left(\sum_{j=1}^{N} p_{j}-q\right) \\
& =\sum_{N=2}^{\infty} \frac{v^{N}}{N !} \Omega_{N}(q),
\end{aligned}
$$

and the normalized single-particle distribution in the c.m. system is

$\frac{2 p_{0}}{\sigma_{\text {tot }}} \frac{d^{3} \sigma}{d^{3} p}=v f\left(p_{T}\right) \frac{\Omega(Q-p)}{\Omega(Q)}$,

with $Q=(\sqrt{s}, \mathbf{0}) ; \quad Q^{2}=s$.

For asymptotic energies the average multiplicity behaves as

$\langle N\rangle \underset{s \rightarrow \infty}{\longrightarrow} v \ln \frac{s}{m^{2}}$ 
and the inclusive single particle distribution reaches its scaling limit

$\frac{1}{\sigma_{\text {tot }}} \frac{d^{2} \sigma}{d x d p_{T}^{2}} \approx \frac{\pi v}{x} f\left(p_{T}\right)(1-x)^{v-1} \equiv D_{\infty}\left(x, p_{T}\right)$,

where $x=2 p_{0} / \sqrt{s}$. The convergence to the scaling limit is, however, rather slow.

For finite energies $(\sqrt{s} \gtrsim 10 \mathrm{GeV})$, a very accurate approximation for the normalized single particle spectrum was given by De Groot [16]

$\frac{1}{\sigma_{\mathrm{tot}}} \frac{d^{2} \sigma}{d x d p_{T}^{2}} \approx D_{\infty}\left(x, p_{T}\right)$.

$\cdot(1-x)^{-v / \bar{n}(s)} \cdot \exp \left[-p_{T}^{2}(1-x)^{-v / \bar{n}(s)} /\left\langle p_{T}^{2}\right\rangle_{A} \bar{n}(s)\right]$,

where $\left\langle p_{T}^{2}\right\rangle_{A}$ is the asymptotic average $p_{T}^{2}$, i.e.

$\left\langle p_{T}^{2}\right\rangle_{A}=\pi \int_{0}^{\infty} f\left(p_{T}\right) p_{T}^{3} d p_{T}$

The quantity $\bar{n}(s)$ approaches asymptotically the average multiplicity $\langle N\rangle$ and is given in Ref. 16 . The correction factors in formula (7) show that the average $p_{T}$ at finite energies will reach its asymptotic value only slowly.

In our actual calculations the transversemomentum cutoff for the pions was taken to be

$f\left(p_{T}\right)=\frac{\lambda^{2}}{\pi} \exp \left(-\lambda p_{T}\right)$

The model depends only on the two parameters $v$ and $\lambda$. Their values were fixed by requiring $\left\langle p_{T}\right\rangle=336 \mathrm{MeV}$ and $\langle N\rangle=16.8$ at $\sqrt{s}=17 \mathrm{GeV}$ :

$\lambda=4.5 \mathrm{GeV}^{-1}, v=4.65$.

The phase-space volumes were then evaluated with the Fourier transform method [17] for $\sqrt{s}=2.5-$ $30 \mathrm{GeV}$ and checked against formula (7) in the region $\sqrt{s}>10 \mathrm{GeV}$.

\section{Results and Discussion}

Within the framework of QCD, scaling violations of fragmentation functions are expressed by the $Q^{2}$ evolution of their moments [5]

$M_{n}\left(Q^{2}\right)=\int_{0}^{1} d x x^{n-1} D\left(x, Q^{2}\right)$.

In our model, the fragmentation function is given by

$D\left(x, Q^{2}\right)=\frac{1}{\sigma_{\text {tot }}} \frac{d \sigma}{d x}\left(x, Q^{2}\right)$.

At finite energies, the choice of the scaling variable $x$ is not unique. Instead of choosing $x=2 p_{0} / \sqrt{s}$, one might use $x_{p}=2 p / \sqrt{s}$ or $x_{\|}=2 p_{\|} / \sqrt{s}$. We have plotted in Figs. 1 and 2 the natural logarithm of the first nontrivial moments for the variables $x$ and $x_{\|}$, respectively, and their asymptotic limits, which are

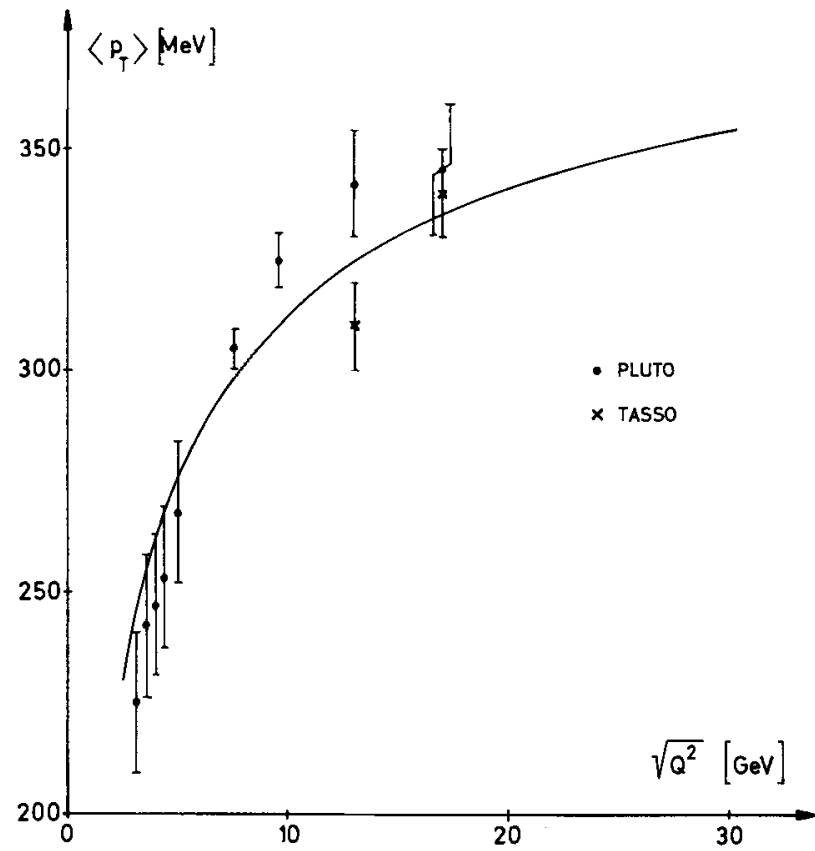

Fig. 3. Average transverse momentum of secondaries versus $\sqrt{Q^{2}}$ from the UJM. Experimental points are from PLUTO [18] and TASSO [7]

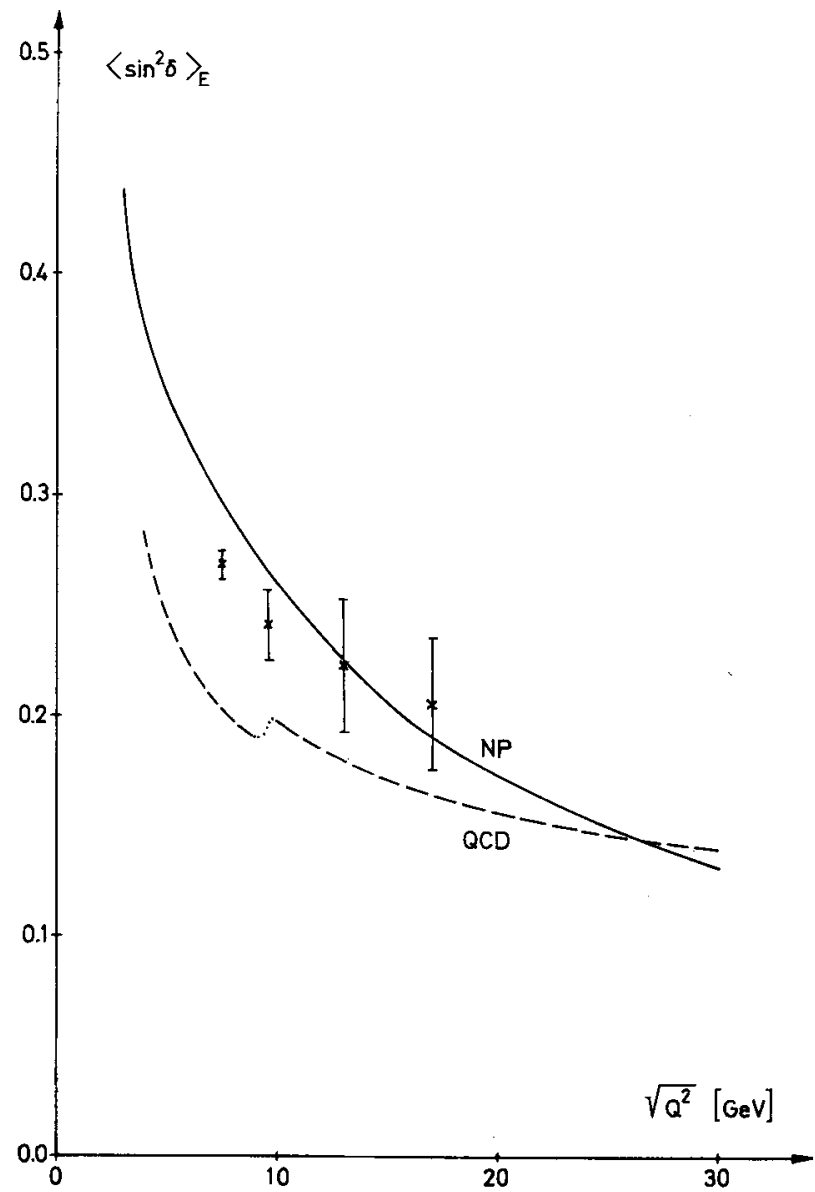

Fig. 4. $\left\langle\sin ^{2} \delta\right\rangle_{E}$ from the UJM (solid line) and QCD (dashed line) with $A^{2}=.5 \mathrm{GeV}^{2}$ and $N_{F}$ varying from 4 to 5 . The experimental points from PLUTO [20] refer to charged particles only 


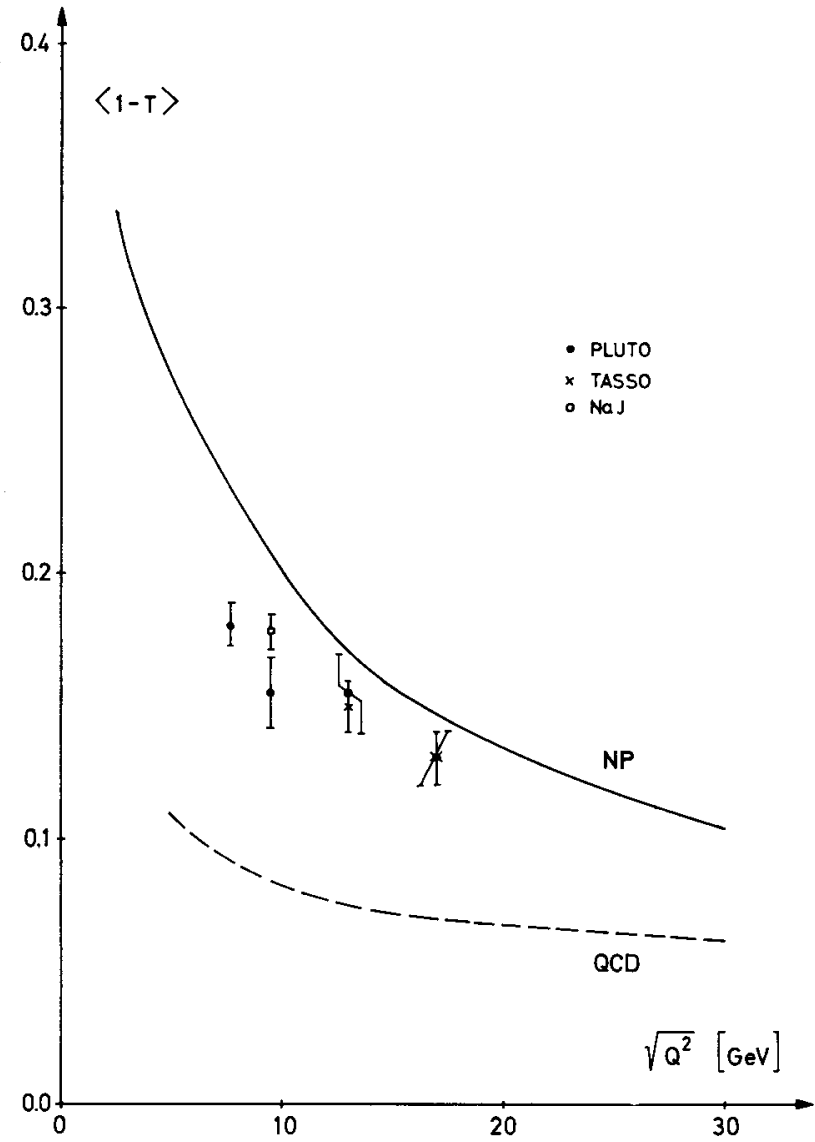

Fig. 5. Average thrust, plotted as $\langle 1-T\rangle$ versus $\sqrt{Q^{2}}$. Plotted are the UJM (Eq. (14), solid curve) and QCD predictions [21] (dashed curve). Experimental points are from PLUTO [20], TASSO [7], and the NaJ detector at DESY [20]

obtained from Eq. (6). All moments show a substantial $Q^{2}$-dependence between $Q^{2}=(2.5 \mathrm{GeV})^{2}$ and $900 \mathrm{GeV}^{2}$. To get an idea about the scale breaking predicted by QCD, we show the $Q^{2}$-evolution of the moments of the non-singlet fragmentation function [5]. They were fixed at $Q_{0}^{2}=(2.5 \mathrm{GeV})^{2}$ to coincide with our model. The $Q C D$ variation turns out to be of the same order as our non-perturbative estimate! We convinced ourselves that the inclusion of singlet terms does not qualitatively change this situation. In view of the very late scaling found for the nonperturbative model, it therefore seems very hard to test perturbative $Q C D$ via moment analyses within this $Q^{2}$ region [6].

The slow approach to asymptotia of the UJM can be seen as well in the $p_{T}$-distribution of secondaries. Figure 3 shows $\left\langle p_{T}\right\rangle$ as a function of $\sqrt{Q}^{2}$. We observe that the asymptotic limit

$\left\langle p_{T}\right\rangle_{\infty}=\frac{2}{\lambda}=444 \mathrm{MeV}$

is by far not reached at $\sqrt{Q^{2}}=30 \mathrm{GeV}$, while the region below $10 \mathrm{GeV}$ exhibits clearly the opening of

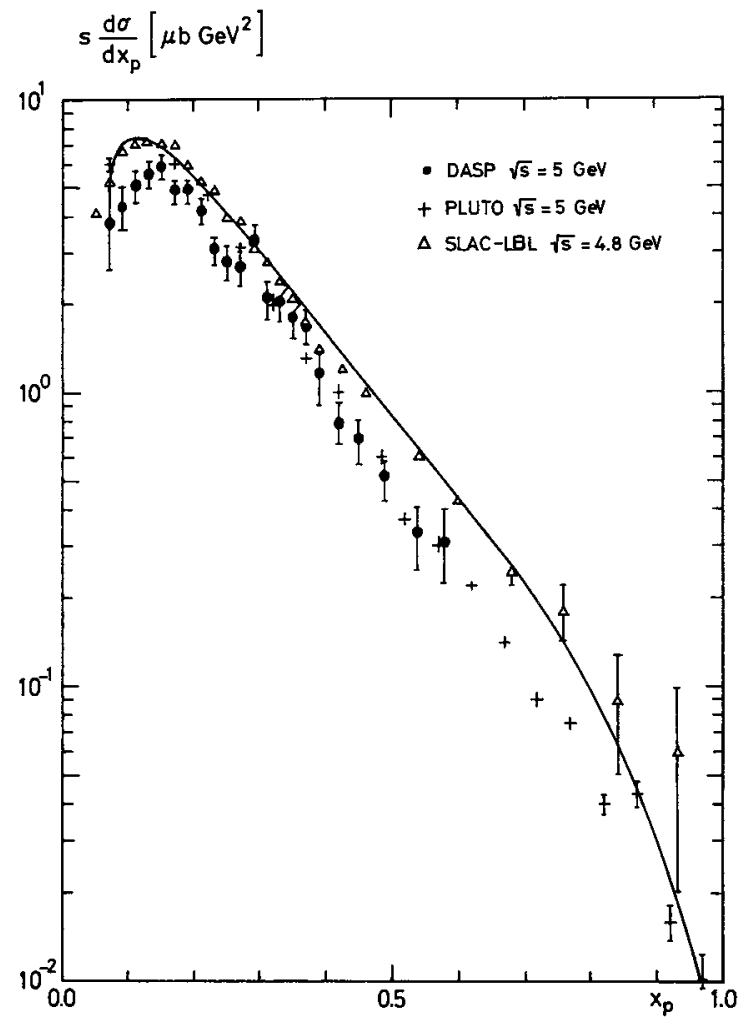

Fig. 6. Comparison of the shape of the (arbitrarily normalized) UJM prediction with charged hadron distributions observed around $5 \mathrm{GeV}$. The variable $x_{p}$ is defined by $x_{p}=2 p / \sqrt{Q^{2}}$. Data as quoted in Ref. 7

phase-space. In the region between 10 and $30 \mathrm{GeV}$, on the other hand, which corresponds roughly to ISR energies (if leading particle effects are subtracted), the value of $\left\langle p_{T}\right\rangle$ lies in the range $335 \pm 20 \mathrm{MeV}$. As far as the experimental points $[7,18]$ in Fig. 3 are concerned, it should be remembered that the observed events at $\sqrt{Q}^{2} \lesssim 5 \mathrm{GeV}$ are very close to isotropy and that the sphericity minimization procedure used to determine $\left\langle p_{T}\right\rangle$ experimentally tends to underestimate the average $p_{T}$.

A popular quantity [11] to determine the QCD jet profile is the energy-weighted mean

$\left\langle\sin ^{2} \delta\right\rangle_{E} \equiv \frac{1}{\sqrt{Q^{2}}} \frac{1}{\sigma_{\text {tot }}} \int d^{3} p \frac{p_{T}^{2}}{p^{2}} p_{0} \frac{d^{3} \sigma}{d^{3} p}$.

As can be seen in Fig. 4, the non-perturbative model dominates the perturbative QCD effects

$\left\langle\sin ^{2} \delta\right\rangle_{E, \mathrm{QCD}}=\frac{24}{\left(33-2 N_{f}\right) \ln Q^{2} / \Lambda^{2}}$

with $\Lambda^{2}=0.5 \mathrm{GeV}^{2}$. The same conclusion has been reached by Steiner [19], who assumed a specific form of angular scaling for the energy flow distri- 


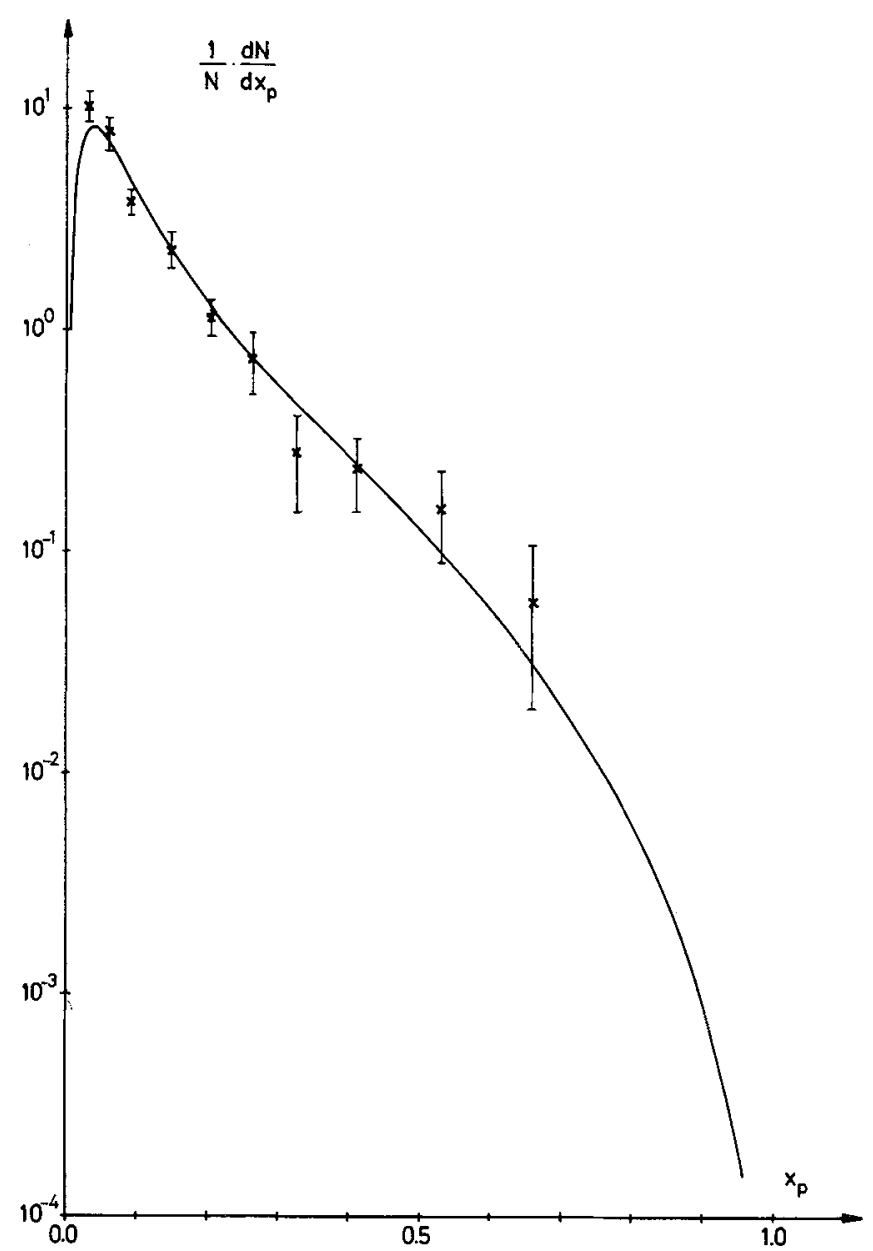

Fig. 7. One particle spectrum, $1 / N d N / d x_{p}$ from the UJM at $17 \mathrm{GeV}$. Data points refer to charged particles measured at TASSO [7]. $x_{p}$ as in Fig. 6

bution. The data points from the PLUTO group [20] contained in Fig. 4 refer to charged tracks only. They are in reasonable agreement with our curve.

The chance to observe QCD effects from average thrust is even worse (see Fig. 5). To a good approximation $\langle T\rangle$ can be easily calculated from inclusive observables

$\langle T\rangle \approx \frac{\left\langle\left|p_{\|}\right|\right\rangle\langle N\rangle}{\sqrt{Q^{2}}}$.

The QCD prediction for $\langle 1-T\rangle$ in Fig. 5 was taken from Ref. 21. In contrast to the optimistic expectation expressed in this reference, we have no hope that measurements of $\langle T\rangle$ will reveal QCD effects below $30 \mathrm{GeV}$.

So far we have only considered mean values. One might think that our trivial two-parameter model is too simple to explain the inclusive distributions as well and therefore should not be seriously discussed.

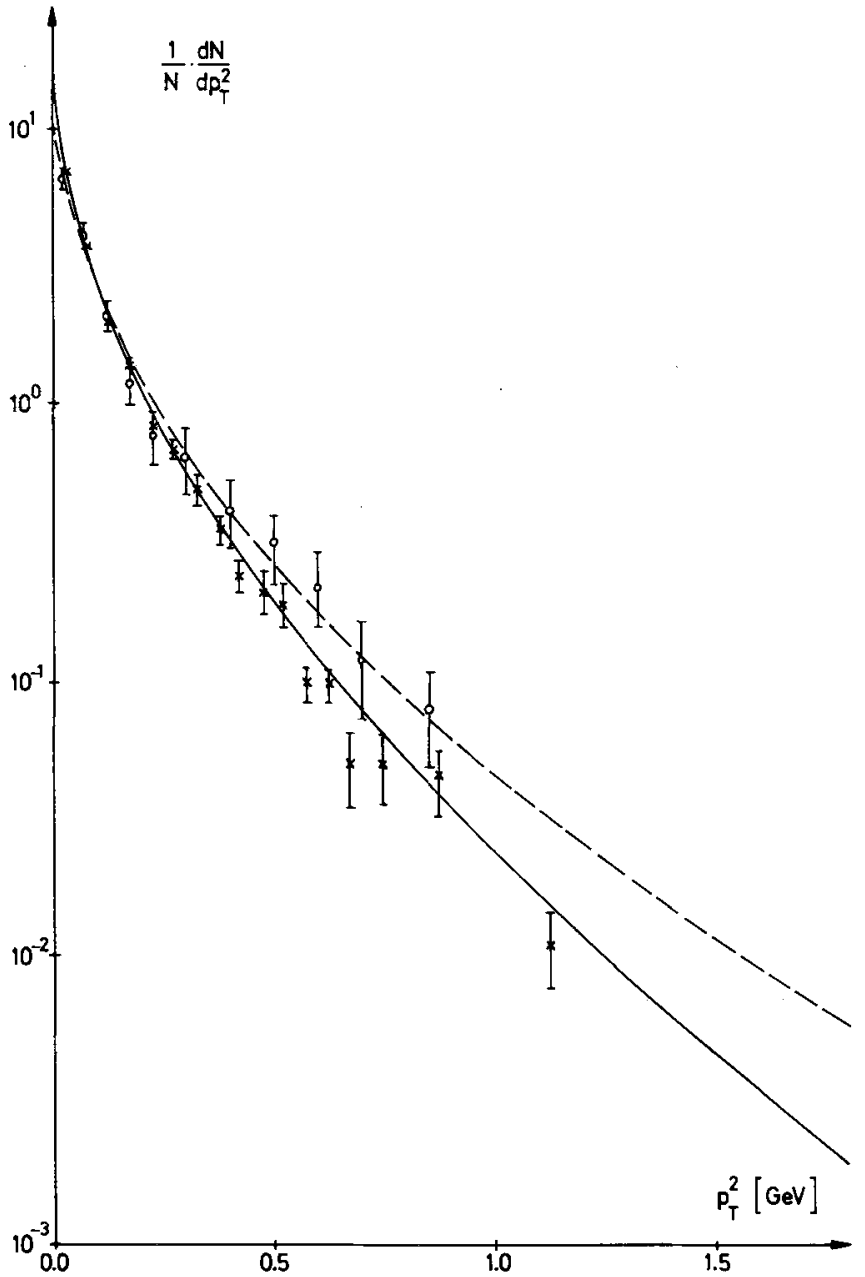

Fig. 8. Transverse distribution, $1 / N d N / d p_{T}^{2}$, versus $p_{T}^{2}$ at $\sqrt{Q^{2}}=$ $17 \mathrm{GeV}(--)$ and $7.5 \mathrm{GeV}(--)$. Data points refer to charged secondaries measured at $17 \mathrm{GeV}(0)$ and $7.7 \mathrm{GeV}(x)$ by PLUTO [20]

However, as can be seen from Figs. 6, 7 and 8, both the $x_{p^{-}}$and $p_{T}^{2}$-distributions are in embarrassing agreement with experiment.

\section{Conclusions}

We conclude that perturbative QCD and subasymptotic non-perturbative scale breaking effects at present storage ring energies are expected to be of the same order of magnitude and similar structure. Hence it will be difficult to separate them from each other. However, the precision of available secondary hadron spectra measured from $e^{+} e^{-}$annihilation is not at all sufficient to detect any scaling violation.

Furthermore, it was demonstrated that QCD infrared-safe global quantities like $\langle 1-T\rangle$ and $\left\langle\sin ^{2} \delta\right\rangle_{E}$, contrary to earlier hopes $[10,11]$, at presently accessible storage ring energies are not sensitive enough to extract information about hard gluon bremsstrahlung. 
It is obvious that such non-perturbative scale breaking effects of at least similar size are present in quark fragmentation from deep inelastic collisions at SPS and FNAL and will substantially affect the proposed tests of factorization breaking from next to leading order corrections [13].

We do not claim that the uncorrelated jet model is the ultimate wisdom to understand jet physics but it is certainly sensible and legitimate to use it in order to sharpen ones eyes for the dynamics of nonperturbative jet development. A prejudice for cascade models with precocious scaling on the other hand might be very misleading.

The hope remains that more detailed and more accurate data will provide us with unambiguous signals in favour of QCD.

Acknowledgements. We thank E.H. de Groot, R. Odorico, N. Sakai and $\mathrm{H}$. Satz for interesting discussions and $\mathrm{H}$. Meyer for explaining to us the experimental implications at PETRA. One of us (J.D.) would like to thank the Physics Department of the Wuppertal University for the kind hospitality extended to him.

\section{References}

1. See e.g. R.D. Field: Plenary Talk presented at the 19th International Conference on High Energy Physics, Tokyo 1978, and references quoted therein

2. See e.g. W. Marciano, H. Pagels: Phys. Reports 36C, 137 (1978)

3. F. Niedermayer: Nucl. Phys. B79, 355 (1974);

B. Anderson, G. Gustafson, C. Peterson: Nucl. Phys. B135, 273 (1978); Z. Physik C 1, 105 (1979);
R.D. Field, R.P. Feynman: Nucl. Phys. B136, 1 (1978);

E.H. de Groot, J. Engels: Z. Physik C 1, 51 (1979)

4. G. Altarelli, G. Parisi : Nucl. Phys. B126, 298 (1977)

5. T. Uematsu: Phys. Lett. 79B, 97 (1978);

J.F. Owens: Phys. Lett. 76B, 85 (1978)

6. D.H. Perkins: Lecture presented at the Rutherford Laboratory X-mas Theoretical Physics Meeting, 1978, Oxford Univ. Preprint 2/79;

N. Schmitz, Talk presented at the Frühjahrstagung der Deutschen Physikalischen Gesellschaft, Sektion Hochenergiephysik Bonn 1979;

D.R.O. Morrison, Talk presented at the 1979 Moriond Meeting

7. Tasso-Collaboration, R. Brandelik et al.: Desy preprint 79/14

8. R. Baier, J. Engels, H. Satz, K. Schilling: Nuovo Cimento A28, 455 (1975)

9. J. Ellis, M.K. Gaillard, G. Ross : Nucl. Phys. B111, 253 (1976)

10. A. de Rujula, J. Ellis, E.G. Floratos, M.K. Gaillard: Nucl. Phys. B138, 387 (1978);

E. Fahri: Phys. Rev. Lett. 39, 1587 (1978);

H. Georgi, M. Machacek: Phys. Rev. Lett. 39, 1237 (1978)

11. C.L. Basham, L.S. Brown, S.D. Ellis, S.T. Love: Phys. Rev. D17, 2298 (1978)

12. G. Kramer, G. Schierholz: Desy preprint $78 / 62$;

G. Kramer, G. Schierholz, J. Willrodt: Desy preprint 78/36

13. N. Sakai: CERN preprint TH-2641; R. Baier, K. Fey: Bielefeld preprint BI-TP 79/11

14. L. van Hove: Rev. Mod. Phys. 36, 655 (1964); A. Krzywicki: Nuovo Cimento 32, 1067 (1964)

15. J. Engels, H. Satz: Phys. Rev. D17, 3015 (1978)

16. E.H. de Groot: Inclusive Distributions at Subasymptotic Energies in the Uncorrelated Jet Model, Bielefeld preprint 1977 (unpublished)

17. J. Engels, J. Fleischer: J. Comput. Phys. 23, 200 (1977)

18. Pluto-Collaboration, Ch. Berger et al.: Desy preprint $78 / 39$ and $\mathrm{H}$. Meyer, private communication

19. F. Steiner: Desy preprint $78 / 59$

20. G. Zech: Review given at the XIVth Rencontre de Moriond 1979, Univ, of Siegen preprint SI 79-2;

21. A. de Rujula: Invited Paper, 19th International Conference on High Energy Physics, Tokyo 1978 